\title{
Versatility and exploratory psychometric properties of the Impulsive/ Premeditated Aggression Scale (IPAS): A review
}

\author{
Ana Rita $\mathrm{Cruz}^{\mathrm{a}, *}$, Andreia de Castro-Rodrigues ${ }^{\mathrm{a}, \mathrm{b}}$, Brian Rundle ${ }^{\mathrm{c}}$, Ioannisely Berrios-Torres ${ }^{\mathrm{d}}$, \\ Rui Abrunhosa Gonçalves ${ }^{\mathrm{a}}$, Fernando Barbosa ${ }^{\mathrm{e}}$, Matthew S. Stanford ${ }^{\mathrm{f}}$ \\ ${ }^{\text {a }}$ CIPsi (Centre of Research in Psychology), School of Psychology, University of Minho, Campus de Gualtar, 4710-057 Braga, Portugal \\ ${ }^{\mathrm{b}}$ Faculty of Law, University Lusíada North (Porto), Rua Dr. Lopo de Carvalho, 4369-006 Porto, Portugal \\ ${ }^{\mathrm{c}}$ Department of Neurology, David Geffen School of medicine, University of California Los Angeles, United States of America \\ ${ }^{\mathrm{d}}$ Behavioral Sciences Research Institute, Medical Sciences Campus, University of Puerto Rico, Puerto Rico \\ ${ }^{\mathrm{e}}$ Laboratory of Neuropsychophysiology, University of Porto, Rua Alfredo Allen, 4200-135 Porto, Portugal \\ ${ }^{\mathrm{f}}$ Hope and Healing Institute, Houston, TX, United States of America
}

\section{A R T I C L E I N F O}

\section{Keywords:}

IPAS versatility

Psychometric properties

Impulsive aggression

Premeditated aggression

\begin{abstract}
A B S T R A C T
Aggression has different conceptualizations and can be behaviorally expressed in diverse ways. Designed to evaluate impulsive and premeditated forms of aggression, the Impulsive/Premeditated Aggression Scale (IPAS; Stanford et al., 2003) is a 30 item self-report questionnaire. The aim of the present study was to explore IPAS versatility in different psychological settings by reviewing and examining the exploratory psychometric properties of the IPAS impulsive and premeditated subscales, across different samples and cultural backgrounds. Fifty-two articles including demographic or psychometric information (internal consistency, factor analysis, validity, reliability) were retrieved. It is suggested that the IPAS is reliable across different cultures, samples and scoring techniques. The two subscales (Impulsive and Premeditated) show acceptable internal consistency. Also, IPAS factors seem to be constant both in clinical and non-clinical samples. The IPAS appears to be a clinically useful instrument for differentiating between subtypes of aggressive behavior, to support risk assessment evaluations, pretrial decisions and better treatment and rehabilitation strategies in offenders and clinical relevant samples.
\end{abstract}

\section{Introduction}

Aggression is a multifaceted and heterogeneous construct but there is little consensus on its definition. Some authors have attempted to define aggression according to the consequences of the aggressive action, such as the behavioral intention of harming or controlling another for one's own needs and to the detriment of others, where the perpetrator is willing to cause harm and the target is motivated to avoid harm (Bushman \& Anderson, 2001). This construct is widely studied in psychological sciences, has different conceptualizations, and can be behaviorally expressed in very different ways (Parrott \& Giancola, 2007).

Despite the diversity of aggressive behavior, aggression can be classified into an (a) emotionally charged, poorly controlled "impulsive" type or (b) a planned, unemotional "premeditated" type (Conner, Houston, Sworts, \& Meldrum, 2007). Impulsive aggression is often associated with affective, impetuous and reactive behavior, while premeditated aggression is associated with planned, deliberate, intentional and proactive behavior (Parrott \& Giancola, 2007; Volavka, 1999). These classifications are considered as the primary forms of aggression (Stanford et al., 2003), representing the natural heterogeneous scheme of aggressive behavior. They seem to discriminate different typologies of aggression, either in terms of phenomenology or neurobiology (Barratt, Stanford, Felthous, \& Kent, 1997; Houston \& Stanford, 2006; Mathias et al., 2007; Meloy, 2006). The primary difference between impulsive and premeditated aggression is the amount of motivation and behavioral control exhibited during the aggressive incident (Stanford et al., 2003; Stanford, Houston, \& Baldridge, 2008). Impulsivity can be defined as a predisposition for rapid and unplanned reactions to external or internal stimuli without considering the negative consequences of these reactions in the person or others (Moeller,

\footnotetext{
* Corresponding author at: Escola de Psicologia, Universidade do Minho-Campus de Gualtar, 4710-057 Braga, Portugal.

E-mail addresses: anaritapcruz@gmail.com (A.R. Cruz), andreiagtcr@gmail.com (A.d. Castro-Rodrigues), brian.rundle@oswego.edu (B. Rundle), ioannisely.berrios@upr.edu (I. Berrios-Torres), rabrunhosa@psi.uminho.pt (R.A. Gonçalves), fbarbosa@fpce.up.pt (F. Barbosa), mstanford@hopeandhealingcenter.org (M.S. Stanford).
} 
Barratt, Dougherty, Schmitz, \& Swann, 2001). The individual expressing impulsive aggression, either verbal or physical, fails to inhibit impulses, which results in aggressive outbursts without any type of regulation or control (Raine et al., 1998). Meanwhile, premeditated aggression is characterized by a planned and pre-established behavior, motivated by external rewards. This subtype of aggressive behavior presupposes that a person is proactive, instrumental, predatory and controlled (Mathias et al., 2007). It requires no anger or provocation, and can be oriented towards the possession of an object or domination over a human being (Vitaro, Brendgen, \& Tremblay, 2002). Stanford et al. (2003) argued that many aggressive individuals will display both premeditated and impulsive acts of aggression, but one form is usually more predominant.

Distinct research approaches have been proposed to study aggressive behavior, either by self-report scales/questionnaires, projective tests, observational techniques, or laboratory aggression paradigms (Suris et al., 2004). The present work will focus on the use of self-report scales. Although it might carry concerns regarding impression management, and social desirability, efforts to improve the psychometric characteristics of the scales along with their potential to provide rapid access to information make them useful resources to study aggressive behavior. Self-report measures may also provide a standardized point of reference against which to evaluate other sources of data (Meloy, 2006). Understanding the tendency of different individuals to premeditated or impulsive aggression has implications in several domains of psychological intervention, namely over criminals, juveniles with behavior disorders, or/and people with mental disabilities, in terms of risk assessment and definition of clinical intervention needs.

\section{Impulsive Premeditated Aggression Scale (IPAS)}

The Impulsive Premeditated Aggression Scale (IPAS; Stanford et al., 2003 ) is a 30 item self-report instrument designed to assess aggressive behavior. Specifically, it was developed to differentiate between two different patterns of aggression - impulsive and premeditated - in both inpatient and community samples. It enables the classification of aggressive acts in ways which have implications for therapeutic and clinical interventions (Stanford, n.d.). Participants are asked to answer according to the occurrence of aggressive acts in the past six months. Only those who have committed physical or verbal aggression including striking, breaking or throwing objects, or verbal attacking others in that period can complete the scale (Stanford et al., 2003). The answers can be scored dimensionally or categorically. Dimensional scoring consists in summing all items assessed on a 5-point Likert scale (1 - Strongly Disagree, 5 - Strongly Agree), which results in an Impulsive Aggression (IA) and Premeditated Aggression (PA) score. On the other hand, categorical scoring results from a percentage of positive items (valued as 4 - Agree, or 5 - Strongly Agree) for each aggression scale (Stanford, n.d.). Dimensional and categorical scoring consider the characterization of aggression within a continuum (Dougherty et al., 2007). The behavior is then defined as predominantly impulsive or predominantly premeditated.

While originally developed and normed in English, the IPAS has been translated into four other languages. Translations of the IPAS are available in Chinese (Chen, Yang, \& Qian, 2013), Dutch (Kuyck, de Beurs, Barendregt, \& van den Brink, 2013), Portuguese (Azevedo, PaisRibeiro, Coelho, \& Figueiredo-Braga, 2018; Cruz \& Barbosa, 2010), and Spanish (Rodríguez, 2015; Romans et al., 2015).

This research aims to improve the knowledge about the impulsive and premeditated aggressive patterns and to assess IPAS applicability and appropriateness across various cultures, populations and psychological settings, through the analysis of the most referred psychometric properties.

\section{Method}

\subsection{Search strategy}

The literature review included articles written in English and Spanish searched on Pub-med, Psych-Info, and Psych Articles and B-ON. Key terms used to identify the selected articles included "Impulsive and Premeditated Aggression Scale", "impulsive" and "premeditated" aggression. These terms were all written under title, abstract and keywords search options. Articles were further reviewed to identify any remaining references to the IPAS that did not appear in the online database search queries.

Initially, fifty-seven studies were selected. Out of those, five were excluded due to only referencing the IPAS. The 53 studies left were included in this review. Psychometric data for the IPAS was reported in 28 of the 53 articles included.

Each article in use was collected and analysed by three reviewers. An archive of relevant information was created, which included study demographics (e.g., type of sample, age, gender) and psychometric data related to the IPAS within the study sample (e.g., reliability, validity, factorial structure).

\section{Results}

\subsection{IPAS psychometric properties}

\subsubsection{Reliability, internal consistency, and intercorrelations}

Of the 53 studies included in the review, comprising the four translated versions of the instrument, 28 reported the internal consistency based on the computed alpha of their data (the other 25 did not address or reported coefficients from previous studies or normative samples) and/or intercorrelations of the IPAS scales (Table 1). These studies assessed a variety of samples including: community participants (5), aggressive boys (1), adolescents (2), forensic samples (11), college students (4), psychiatric patients (2), veterans (2), and substance use participants (3). Twenty-two studies used both female and male participants, while six evaluated only males, and one evaluated females.

The PA scale evidenced acceptable to high internal consistency with Cronbach's alpha ranging from 0.70 to 0.90 for the scores of the reported samples. The alpha coefficients for the IA scale ranged from 0.37 to 0.93 , but the lower value might be explained by the small sample of the study (Teten, Miller, Bailey, Dunn, \& Kent, 2008). If this value is discarded, internal consistency is satisfactory, ranging from 0.63 to 0.93 .

However, the alpha coefficient is not a property of the test, rather of the scores and should not be considered stable across samples (Rouse, 2007). Since alpha is sample-specific, meta-analytic analysis are required to explore variance in reliability coefficients to allow for comparisons regarding the influence of variables such as culture, language or gender (Rodriguez \& Maeda, 2006; Rouse, 2007; Vassar \& Bradley, 2010). We have conducted a reliability generalization analysis of the IPAS scales based on the procedure described by Rouse (2007) examining alpha coefficients for sample (clinical/forensic or from the community), form of the test (English or translated) and scoring technique (dimensional or categorical). The mean reliability estimates (see Table 2) obtained for PA and IA scales for sample (PA: $t(23)=0.589$, $p=.561, g=0.31$; IA: $t(24)=1.29, p=.208, g=0.567)$, and form of the test (PA: $t(23)=-0.557, p=.583, g=0.158$; IA: $t(24)=-1.49$, $p=.150, g=0.637$ ) did not produce significant differences. However our results demonstrated a marginal difference for IA, though not for $\mathrm{PA}$, regarding scoring technique, with the dimensional scoring technique producing higher reliability, (PA: $t(10)=0.534, p=.605$, $g=0.333$; IA: $t(10)=2.15, p=.057, g=1.40)$. We should also note that our data revealed large effect sizes, although not significant, probably because of the small number of studies analysed. Effect sizes for IA were larger than for PA, suggesting that an increase in 
Table 1

Reliability and intercorrelations among IPAS scales.

\begin{tabular}{|c|c|c|c|c|c|c|}
\hline \multirow[t]{2}{*}{ Studies } & \multirow[t]{2}{*}{ Participants } & \multicolumn{2}{|c|}{ Gender (\%) } & \multirow[t]{2}{*}{ Cronbach's alpha } & \multirow[t]{2}{*}{ Intercorrelation } & \multirow[t]{2}{*}{ Scoring } \\
\hline & & Male & Female & & & \\
\hline Stanford et al., 2003 & 93 physically aggressive psychiatric outpatients & $100 \%$ & - & PA $0.82 ;$ IA 0.77. & $r=-.02^{\mathrm{a}}$ & Categorical \\
\hline Kockler et al., 2006 & $\begin{array}{l}86 \text { participants recruited as a non-random sample of } \\
\text { convenience from a forensic state hospital }\end{array}$ & $85.9 \%$ & $14.1 \%$ & PA 0.72 ; IA 0.81. & $r=0.40^{* *}$ & Categorical \\
\hline Conner et al., 2007 & 121 patients in a methadone maintenance treatment & $49.6 \%$ & $50.4 \%$ & PA 0.75; IA.74 & $r=.018^{\mathrm{d}}$ & Dimensional \\
\hline Derkzen, 2007 & 185 incarcerated offenders & $100 \%$ & - & PA 0.74; IA 0.63; & $r=0.364^{* *}$ & Categorical \\
\hline Dougherty et al., 2007 & $\begin{array}{l}64 \text { Adolescents with conduct disorder and } 37 \text { healthy } \\
\text { controls }\end{array}$ & $48.5 \%$ & $51.5 \%$ & nd & $r=.23^{\mathrm{b}}$ & Categorical \\
\hline Mathias et al., 2007 & 66 adolescents & $63.6 \%$ & $36.4 \%$ & PA 0.78 ; IA 0.82 & nd & nd \\
\hline Haden et al., 2008 & 340 college students & $37.4 \%$ & $62.6 \%$ & PA 0.81 ; IA 0.77 & $r=0.32^{* *}$ & Dimensional \\
\hline Ostrov \& Houston, 2008 & 679 emerging adults & $52 \%$ & $48 \%$ & PA $0.89 ;$ IA 0.88 . & $r=0.70^{*}$ & Dimensional \\
\hline Stanford et al., 2008 & 113 men convicted of domestic violence & $100 \%$ & - & PA 0.86; IA 0.75 & $r=0.50^{* *}$ & Categorical \\
\hline Teten et al., 2008 & 82 veterans & $92.1 \%$ & $7.9 \%$ & PA nd; IA 0.37 & nd & nd \\
\hline $\begin{array}{l}\text { Conner, Swogger, \& Houston, } \\
2009\end{array}$ & 878 patients in substance-dependence treatment programs & $70.8 \%$ & $29.2 \%$ & PA $0.77 ;$ IA 0.73 & nd & nd \\
\hline Gauthier et al., 2009 & 56 adolescents with CD & $61 \%$ & $39 \%$ & nd & $r=.16^{\mathrm{c}}$ & nd \\
\hline Cruz \& Barbosa, 2010 & 91 participants recruited from the community & $27.5 \%$ & $72.5 \%$ & PA 0.76 ; IA 0.75 & nd & $\begin{array}{c}\text { Categorical and } \\
\text { dimensional }\end{array}$ \\
\hline Swogger et al., 2010 & 95 criminal offenders charged with a crime & $74.7 \%$ & $25.3 \%$ & PA 0.86; IA 0.82 & $r=0.51^{* *}$ & nd \\
\hline Teten et al., 2010 & 136 veterans & $100 \%$ & - & PA 0.75 ; IA 0.75 & $r=0.27^{* *}$ & $\begin{array}{l}\text { Categorical and } \\
\text { dimensional }\end{array}$ \\
\hline Lake \& Stanford, 2011 & 87 offenders & - & $100 \%$ & PA 0.82 ; IA 0.71 & $r=0.38^{* *}$ & Categorical \\
\hline Teten Tharp et al., 2011 & 250 psychology students & $14.4 \%$ & $85.6 \%$ & PA 0.73 ; IA 0.74 & $r=0.14^{*}$ & Dimensional \\
\hline Chen et al., 2013 & 290 university students & nd & nd & PA 0.70 ; IA 0.67 & $r=0.057^{\mathrm{a}}$ & nd \\
\hline Kuyck et al., 2013 & 219 prisoners & $68 \%$ & $32 \%$ & PA 0.90; IA 0.93 & $r=.71^{\mathrm{e}}$ & nd \\
\hline Steadham \& Rogers, 2013 & 93 pre and post-trial detainees & $48.4 \%$ & $51.6 \%$ & nd & $r=0.69^{* * *}$ & Categorical \\
\hline Long et al., 2014 & 81 adults in a residential substance use facility & $84 \%$ & $16 \%$ & PA 0.87; IA.86 & $r=0.75^{* *}$ & nd \\
\hline Swing and Anderson, 2014 & 422 undergraduate students & $39 \%$ & $61 \%$ & PA 0.88 ; IA 0.82 & nd & nd \\
\hline Swogger et al., 2014 & 91 adults charged with a crime & $73.6 \%$ & $26.4 \%$ & PA 0.82 ; IA 0.86 & $r=0.57^{* *}$ & nd \\
\hline $\begin{array}{l}\text { Swogger, Walsh, Maisto, \& } \\
\quad \text { Conner, } 2014\end{array}$ & 96 adult criminal offenders & $76 \%$ & $24 \%$ & PA 0.82 ; IA 0.86 & $r=0.59^{* *}$ & Dimensional \\
\hline Rodríguez, 2015 & 953 community adults & $50.3 \%$ & $49.7 \%$ & PA 0.89 ; IA 0.82 & nd & nd \\
\hline Romans et al., 2015 & 163 psychiatric patients & $30.1 \%$ & $69.9 \%$ & PA 0.76; IA.85 & nd & nd \\
\hline Azevedo et al., 2018 & 240 prisoners & $100 \%$ & - & PA 0.88; IA 0.89 & nd & nd \\
\hline \multirow[t]{2}{*}{ Cruz et al., 2019} & 957 community adults & $44.3 \%$ & $55.7 \%$ & PA 0.81 ; IA 0.86 & $r=0.34^{*}$ & Categorical and \\
\hline & 115 prisoners & $100 \%$ & - & PA 0.86 ; IA 0.86 & $r=0.48^{* * *}$ & dimensional \\
\hline
\end{tabular}

Note. nd = no data.

a The IA and PM scales were not significantly intercorrelated.

b $\mathrm{CD}$ group.

c $p>.05$.

d $p=.839$.

e Highly correlated, but no value provided.

* $p<.05$.

*** $p<.01$

${ }^{* * *} p<.001$.

Table 2

Mean reliability (alpha coefficient) and standard deviation for PA and IA for sample, form of the test and scoring technique.

\begin{tabular}{lclcl}
\hline & $n$ & PA & $n$ & IA \\
\hline Non-clinical sample & 11 & $0.80(0.07)$ & $12^{\mathrm{a}}$ & $0.75(0.13)$ \\
Clinical sample & 14 & $0.82(0.06)$ & 14 & $0.81(0.08)$ \\
English version & 17 & $0.81(0.06)$ & $18^{\mathrm{a}}$ & $0.76(0.12)$ \\
Translated version & 8 & $0.82(0.07)$ & 8 & $0.83(0.08)$ \\
Dimensional scoring & 7 & $0.81(0.06)$ & 7 & $0.82(0.06)$ \\
Categorical scoring & 5 & $0.79(0.06)$ & 5 & $0.73(0.07)$ \\
\hline
\end{tabular}

Note:

a The difference on the $n$ is related to a study that only reported IA vales.

observations could produce significant differences across samples and form of the test. Regarding gender, only four studies reported alpha coefficients for both male and female participants, therefore we did not procced to a reliability generalization analysis with those data.

Intercorrelation between the IA and PA scales was not consistent in the literature: 14 studies found a significant positive correlation between the scales (Cruz et al., 2019; Derkzen, 2007; Haden, Scarpa, \& Stanford, 2008; Kockler, Stanford, Nelson, Meloy, \& Sanford, 2006;
Kuyck et al., 2013; Long, Felton, Lilienfeld, \& Lejuez, 2014; Ostrov \& Houston, 2008; Stanford et al., 2008; Steadham \& Rogers, 2013; Swogger, Walsh, Christie, Priddy, \& Conner, 2014; Swogger, Walsh, Houston, Cashman-Brown, \& Conner, 2010; Swogger, Walsh, Maisto, \& Conner, 2014; Teten et al., 2010; Teten Tharp et al., 2011), while four found no significant relationship (Chen et al., 2013; Conner et al., 2007; Gauthier, Furr, Mathias, Marsh-Richard, \& Dougherty, 2009; Stanford et al., 2003), and one a low order correlation (Dougherty et al., 2007). The data demonstrates that the scales are not necessarily independent, suggesting that some individuals may exhibit both premeditated and impulsive aggression.

Sensitivity and specificity of impulsive and premeditated aggression were calculated in a sample of adolescents with conduct disorder, comparing the agreement between a semi-structured aggression interview and the self-report questionnaire. It was demonstrated that in more than $96 \%$ of the individuals evaluated, the proportion of not having the characteristic was correctly identified (Mathias et al., 2007). As for the sensitivity, $60 \%$ of individuals with premeditated aggression and $50 \%$ with impulsive aggression were correctly identified (Mathias et al., 2007). 


\subsubsection{Factor structure}

There were studies that did not investigate IPAS internal structure, and used the original components proposed by Stanford et al. (2003) to compute IA and PA scores (e.g., Bo et al., 2013b; Conner et al., 2007; Derkzen, 2007; Stanford et al., 2008; Teten et al., 2010; Wilson \& Scarpa, 2014) or the revised components (Bo et al., 2013a, 2013b; Cruz \& Barbosa, 2010; Teten Tharp et al., 2011). Based on sample characteristics, some studies addressed item dimensions previously proposed (Gauthier et al., 2009; Steinberg, Sharp, Stanford, \& Tharp, 2013; Swogger et al., 2010; Swogger, Walsh, Christie, et al., 2014).

Within the retrieved studies, ten Principal Components Analyses (PCA) were reported (Azevedo et al., 2018; Chen et al., 2013; Conner et al., 2009; Cruz et al., 2019; Kockler et al., 2006; Mathias et al., 2007; Rodríguez, 2015; Romans et al., 2015; Stanford et al., 2003), along with one Confirmatory Factor Analysis (CFA) (Kuyck et al., 2013). Three studies reported both PCA and CFA (Azevedo et al., 2018; Chen et al., 2013; Rodríguez, 2015). Both IA and PM factors were present in the studies that performed factor analysis. Three studies identified a third factor (Chen et al., 2013; Conner et al., 2009; Romans et al., 2015), but most studies presented a two-factor structure accounting for approximately $30 \%$ of the total variance. The participants were classified as predominantly premeditated or impulsive, with the two-factor solution providing the best theoretical and statistical differentiation of the type of aggressive behavior. Moreover, there were no significant differences in the distribution of IPAS items across dimensions.

See Table 3 for results on the factorial structure of the IPAS.

\subsubsection{Validity}

Since impulsivity and premeditation are important features of human behavior it is not surprising that both have also been tested in relation to measures of personality. We have made a systematic review from the retrieved papers that reported Pearson's correlation coefficients between IPAS and other scales, describing both construct (convergent or discriminant) and concurrent validity. Reported correlations between the IPAS and other behavioral and self-report measures of aggression and personality are shown in Table 4.

Most studies (46.4\%) have tested the construct validity between IPAS and other scales that assessed theoretical and empirical related constructs, such as impulsivity (Chen et al., 2013; Derkzen, 2007; Mathias et al., 2007; Stanford et al., 2003; Steinberg et al., 2013), or aggressive features such as anger and hostility (Chen et al., 2013; Mathias et al., 2007; Stanford et al., 2003; Teten Tharp et al., 2011). Fifteen studies (53.6\%) have used well-known and widely used measures of aggression, such as the BIS-11 and the BPAQ, but have also tested construct validity with other measures that assessed reactive and proactive aggression (e.g., RPQ).
The IA and PA subscales of the IPAS were expected to be significantly, though differently, correlated with aggression related constructs. For example, anger was expected to be more correlated with IA, whereas hostility was expected to be correlated with PA features; these latter were expected to be more related with psychopathy than IA features. In accordance, there was a similar association between the BPAQ subscales and IA and PA, even if the correlation IA-Anger tends to be slightly higher than the PA-Anger association (Rodríguez, 2015; Stanford et al., 2003; Teten et al., 2010; Teten Tharp et al., 2011).

Convergent validity was, therefore, reported between both impulsivity and premeditation, and overt forms of aggressive behaviors, such as anger (e.g., Stanford et al., 2003), frequency of aggression throughout lifetime, and psychopathy factors.

Regarding psychopathy, it was expected that PA would be more correlated with psychopathic features, nevertheless, the reported correlations between IPAS and measures of psychopathy suggest that results could vary in function of the psychopathy instruments (e.g., PCLR, PPI) or the samples (e.g., forensic or clinical) (e.g., Azevedo et al., 2018; Long et al., 2014). From the current review, IA revealed higher convergence with measures of psychopathy than PA. When testing the association of IPAS subscales with emotional regulation difficulties IA was positively correlated with emotional problems, but no significant correlation was found for PA. In line with this, IA revealed to be associated with thought and emotional regulation problems, while PA was associated with personality disturbance, such as psychoticism (Gauthier et al., 2009; Mathias et al., 2007).

In general, reported data on convergent and concurrent validity, although significant, has small to moderate positive correlation values. These results suggest only partial association between the aforementioned variables (psychopathy, aggression, impulsivity and personality characteristics) and both IA and PA.

\subsection{Versatility of the IPAS in different groups}

In the following sections we present the main findings of previous research regarding the applicability of the IPAS to test aggressiveness features in different samples. For that purpose, we describe the main findings of the literature using the IPAS having in mind the adequacy of the scale and the importance of replicating such findings.

\subsubsection{Community samples}

Within the studies that employed the scale using community samples, the majority assessed its characteristics in college settings (Chen et al., 2013; Cruz \& Barbosa, 2010; Haden et al., 2008; Rodríguez, 2015; Steinberg et al., 2013; Swing \& Anderson, 2014), testing the validity of the two-dimensional classification of aggression (e.g., Chen

Table 3

Main results regarding IPAS factorial structure.

\begin{tabular}{|c|c|c|c|c|c|}
\hline \multirow[t]{2}{*}{ Studies } & \multirow[t]{2}{*}{ Sample } & \multirow[t]{2}{*}{ Factorial Method/rotation } & \multicolumn{3}{|c|}{ Original items distribution on observed factors } \\
\hline & & & Factor 1 & Factor 2 & Factor 3 \\
\hline Stanford et al., 2003 & 93 physically aggressive psychiatric outpatients & PCA/varimax & PA & IA & FTRA \\
\hline Kockler et al., 2006 & Forensic state hospital & PCA/varimax & PA & IA & - \\
\hline Mathias et al., 2007 & Adolescents & $\mathrm{PCA} /$ varimax & IA & PA & - \\
\hline Haden et al., 2008 & College students & PCA/varimax & PA & EMA & IA \\
\hline Conner et al., 2009 & Patients in substance-dependence treatment programs & PCA/varimax & PA & IA & Unidentified \\
\hline Chen et al., 2013 & University students & $\mathrm{PCA}$ /varimax and CFA & PA & IA & FC \\
\hline Kuyck et al., 2013 & Prisoners & CFA & PA & IA & - \\
\hline Rodríguez, 2015 & Community adults & PCA/Promax and CFA & PA & IA & - \\
\hline Romans et al., 2015 & Psychiatric patients & PCA/equamax & IA & PA & - \\
\hline Azevedo et al., 2018 & Prisoners & PCA/CFA & IA & PA & - \\
\hline \multirow[t]{2}{*}{ Cruz et al., 2019} & Community adults & PCA/varimax & IA & PA & - \\
\hline & Prisoners & PCA/varimax & IA & PA & - \\
\hline
\end{tabular}

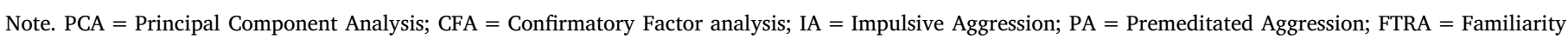
with Target/Remorse/Agitation; EMA = External Motivated Aggression; FC = Feeling of Control. 
Table 4

IPAS reported validity with other scales.

\begin{tabular}{|c|c|c|c|c|c|}
\hline Reported validity & Study & Sample & Scale & IA & PA \\
\hline \multirow[t]{5}{*}{ Construct validity } & \multirow[t]{5}{*}{ Stanford et al., 2003} & \multirow{5}{*}{$\begin{array}{l}\text { Physically aggressive psychiatric outpatients } \\
(n=93)\end{array}$} & BPAQ - Anger & $0.53^{*}$ & 0.16 \\
\hline & & & BPAQ - Hostility & 0.11 & 0.34 \\
\hline & & & BPAQ - Verbal Aggression & 0.20 & $0.32^{*}$ \\
\hline & & & BPAQ - Physical Aggression & $0.30^{*}$ & $0.45^{*}$ \\
\hline & & & BPAQ - Total score & $0.38^{*}$ & $0.51^{*}$ \\
\hline Convergent validity & Derkzen, 2007 & Incarcerated offenders $(n=185)$ & BPAQ - Total score & $0.53^{* *}$ & $0.49^{* * *}$ \\
\hline \multirow[t]{4}{*}{ Construct validity } & \multirow[t]{4}{*}{ Mathias et al., 2007} & \multirow[t]{4}{*}{ Adolescents $(n=66)$} & BPAQ - Anger & $0.35^{*}$ & $0.43^{*}$ \\
\hline & & & BPAQ - Hostility & $0.47^{*}$ & 0.19 \\
\hline & & & BPAQ - Verbal Aggression & 0.20 & $0.54 *$ \\
\hline & & & BPAQ - Physical Aggression & 0.14 & $0.47^{*}$ \\
\hline \multirow[t]{2}{*}{ Construct validity } & \multirow[t]{2}{*}{ Teten et al., 2010} & \multirow[t]{2}{*}{ Veterans $(n=136)$} & BPAQ - Anger & $0.53^{*}$ & $0.21^{*}$ \\
\hline & & & BPAQ - Hostility & $0.31^{*}$ & $0.32 *$ \\
\hline \multirow[t]{4}{*}{ Convergent validity } & \multirow[t]{4}{*}{ Teten Tharp et al., 2011} & \multirow[t]{4}{*}{ Psychology students $(n=250)$} & BPAQ - Anger & $0.26^{*}$ & $0.23^{*}$ \\
\hline & & & BPAQ - Hostility & $0.28^{*}$ & $0.18^{*}$ \\
\hline & & & BPAQ - Verbal Aggression & $0.15^{*}$ & $0.20^{*}$ \\
\hline & & & BPAQ - Physical Aggression & $0.15^{*}$ & $0.30^{*}$ \\
\hline Concurrent validity & Chen et al., 2013 & University students $(n=290)$ & BPAQ - Anger & 0.09 & $0.24^{* *}$ \\
\hline & & & BPAQ - Hostility & 0.05 & $0.26^{* *}$ \\
\hline & & & BPAQ - Verbal Aggression & -0.02 & $0.12^{*}$ \\
\hline & & & BPAQ - Physical Aggression & -0.02 & $0.31^{* *}$ \\
\hline Convergent validity & Rodríguez, 2015 & Adults from the community $(n=953)$ & BPAQ - Anger & $0.45^{* *}$ & $0.39^{* *}$ \\
\hline & & & BPAQ - Hostility & $0.39 * *$ & $0.36^{* *}$ \\
\hline & & & BPAQ - Verbal Aggression & $0.38^{* *}$ & $0.34^{* *}$ \\
\hline & & & BPAQ - Physical Aggression & $0.49^{* *}$ & $0.66^{* *}$ \\
\hline Construct validity & Stanford et al., 2003 & $\begin{array}{l}\text { Physically aggressive psychiatric outpatients } \\
(n=93)\end{array}$ & BIS-11 Total score & $0.21^{*}$ & $0.38^{*}$ \\
\hline & Derkzen, 2007 & Incarcerated offenders $(n=185)$ & BIS-11 Total score & $0.38^{* *}$ & $0.39^{* * *}$ \\
\hline Construct validity & Mathias et al., 2007 & Adolescents $(n=66)$ & BIS-11 Total score & $0.39^{*}$ & $0.26^{*}$ \\
\hline Construct validity & Chen et al., 2013 & University students $(n=290)$ & BIS-11 Attentional impulsiveness & $0.15^{*}$ & 0.19 ** \\
\hline & & & BIS-11 Motor impulsiveness & $0.21^{* *}$ & 0.08 \\
\hline & & & BIS-11 Nonplanning impulsiveness & $0.12^{*}$ & 0.05 \\
\hline Construct validity & Steinberg et al., 2013 & Undergraduate students $(n=1178)$ & BIS-11 Total score & $0.46^{*}$ & $0.20^{*}$ \\
\hline Convergent validity & Rodríguez, 2015 & Adults from the community $(n=953)$ & BIS-11 Attentional impulsiveness & $0.21^{* *}$ & $0.31^{* *}$ \\
\hline & & & BIS-11 Motor impulsiveness & $0.39^{* *}$ & $0.42^{* *}$ \\
\hline & & & BIS-11 Nonplanning impulsiveness & $0.14 * *$ & $0.25^{* *}$ \\
\hline Convergent validity & Azevedo et al., 2018 & Prisoners $(n=240)$ & BIS-11 Attentional impulsiveness & $0.14^{*}$ & $0.18^{*}$ \\
\hline & & & BIS-11 Motor impulsiveness & $0.26^{* *}$ & $0.27^{* * *}$ \\
\hline & & & BIS-11 Nonplanning impulsiveness & $0.15^{*}$ & 0.06 \\
\hline & & & BIS-11 Total score & $0.21^{* *}$ & $0.20^{* *}$ \\
\hline Construct validity & Steinberg et al., 2013 & Undergraduate students $(n=1178)$ & BIS-Brief & $0.37^{*}$ & $0.17^{*}$ \\
\hline Convergent validity & Teten Tharp et al., 2011 & Psychology students $(n=250)$ & PPI Total score & $0.13^{*}$ & $0.14^{*}$ \\
\hline Convergent validity & Long et al., 2014 & Adults in a residential substance use facility & PPI Fearlessness & $0.40^{*}$ & $0.33^{*}$ \\
\hline & & $(n=81)$ & PPI Impulsive Nonconformity & $0.28^{*}$ & 0.32 \\
\hline & & & PPI Blame Externalization & $0.24 *$ & 0.13 \\
\hline & & & PPI Childlike Nonconformity & $0.23^{*}$ & 0.19 \\
\hline & & & PPI Machiavellian Egocenticity & $0.38^{*}$ & $0.34 *$ \\
\hline Divergent validity & Azevedo et al., 2018 & Prisoners $(n=240)$ & PCL-R Interpersonal & -0.14 & $0.24 *$ \\
\hline & & & PCL-R Affective & -0.14 & $0.16^{*}$ \\
\hline & & & PCL-R Lifestyle & -0.07 & $0.22^{*}$ \\
\hline & & & PCL-R Antisocial & -0.07 & $0.21^{*}$ \\
\hline & & & PCL-R Total score & 0.01 & $0.29 * *$ \\
\hline Construct validity & Stanford et al., 2003 & $\begin{array}{l}\text { Physically aggressive psychiatric outpatients } \\
(n=93)\end{array}$ & EPQ Neuroticism & $0.24^{*}$ & $0.40^{*}$ \\
\hline & & & EPQ Extraversion & -0.01 & -0.22 \\
\hline & & & EPQ Psychoticsm & 0.03 & $0.57^{*}$ \\
\hline Construct validity & Mathias et al., 2007 & Adolescents $(n=66)$ & EPQ Neuroticism & $0.54^{*}$ & 0.20 \\
\hline & & & EPQ Psychoticsm & $0.33^{*}$ & $0.43^{*}$ \\
\hline Construct validity & Stanford et al., 2003 & Physically aggressive psychiatric outpatients & LHA Aggression & $0.24^{*}$ & $0.30^{*}$ \\
\hline & & $(n=93)$ & LHA Antisocial Behavior & -0.09 & $0.36^{*}$ \\
\hline & & & LHA Self-directed aggression & -0.01 & $0.27^{*}$ \\
\hline & & & LHA Total score & 0.08 & $0.41^{*}$ \\
\hline Construct validity & Stanford et al., 2003 & Physically aggressive psychiatric outpatients & STAXI Trait Anger & $0.40^{*}$ & $0.41^{*}$ \\
\hline & & $(n=93)$ & STAXI Angry Temperament & $0.37^{*}$ & $0.37^{*}$ \\
\hline & & & STAXI Angry Reaction & $0.31^{*}$ & $0.31^{*}$ \\
\hline & & & STAXI Anger Expression & $0.25^{*}$ & $0.38^{*}$ \\
\hline & & & STAXI Anger In & $0.26^{*}$ & $0.39 *$ \\
\hline & & & STAXI Anger Out & $0.26^{*}$ & $0.29^{*}$ \\
\hline & & & STAXI Anger Control & -0.42 & -0.09 \\
\hline Discriminant validity & Gauthier et al., 2009 & Adolescents with CD $(n=56)$ & NEO-PI-R Neuroticism & $0.59 *$ & 0.16 \\
\hline & & & NEO-PI-R Agreeableness & -0.06 & $0.27^{*}$ \\
\hline
\end{tabular}


Table 4 (continued)

\begin{tabular}{|c|c|c|c|c|c|}
\hline Reported validity & Study & Sample & Scale & IA & PA \\
\hline \multirow[t]{6}{*}{ Construct validity } & \multirow[t]{6}{*}{ Mathias et al., 2007} & \multirow[t]{6}{*}{ Adolescents $(n=66)$} & YSR Aggressive behavior & $0.30^{*}$ & $0.30^{*}$ \\
\hline & & & YSR Social Problems & $0.45^{*}$ & 0.21 \\
\hline & & & YSR Thought Problems & $0.46^{*}$ & 0.22 \\
\hline & & & YSR Attention Problems & $0.47^{*}$ & 0.14 \\
\hline & & & YSR Somatic Complaints & $0.51^{*}$ & $0.26^{*}$ \\
\hline & & & YSR Anxious & $0.41^{*}$ & 0.14 \\
\hline \multirow[t]{4}{*}{ Convergent and discriminant validity } & \multirow[t]{4}{*}{ Teten Tharp et al., 2011} & \multirow[t]{4}{*}{ Psychology students $(n=250)$} & RPQ Proactive Aggression raw score & $0.20^{*}$ & $0.27^{*}$ \\
\hline & & & $\begin{array}{l}\text { RPQ Proactive Aggression residualized } \\
\text { score }\end{array}$ & 0.07 & $0.16^{*}$ \\
\hline & & & RPQ Reactive Aggression raw score & $0.27^{*}$ & $0.25^{*}$ \\
\hline & & & $\begin{array}{l}\text { RPQ Reactive Aggression residualized } \\
\text { score }\end{array}$ & $0.19 *$ & 0.12 \\
\hline \multirow[t]{2}{*}{ Convergent validity } & \multirow[t]{2}{*}{ Teten Tharp et al., 2011} & \multirow[t]{2}{*}{ Psychology students $(n=250)$} & PAI Verbal Aggression & $0.17^{*}$ & $0.16^{*}$ \\
\hline & & & PAI Physical Aggression & $0.36^{*}$ & $0.13^{*}$ \\
\hline Discriminant validity & Long et al., 2014 & $\begin{array}{l}\text { Adults in a residential substance use facility } \\
(n=81)\end{array}$ & DERS & $0.38^{*}$ & 0.11 \\
\hline \multirow[t]{4}{*}{ Discriminant validity } & \multirow[t]{4}{*}{ Ostrov \& Houston, 2008} & \multirow[t]{4}{*}{ Emerging adults $(n=679)$} & SRASBM Proactive Relational Aggression & 0.22 & 0.34 \\
\hline & & & SRASBM Reactive Relational Aggression & $0.21 *$ & $0.28^{*}$ \\
\hline & & & SRASBM Proactive Physical Aggression & $0.23^{*}$ & $0.33^{*}$ \\
\hline & & & SRASBM Reactive Physical Aggression & $0.23^{*}$ & $0.29 *$ \\
\hline \multirow[t]{2}{*}{ Discriminant validity } & \multirow[t]{2}{*}{ Ostrov \& Houston, 2008} & \multirow[t]{2}{*}{ Emerging adults $(n=679)$} & PDQ-4 Borderline Personality & $0.40^{*}$ & $0.31^{*}$ \\
\hline & & & PDQ-4 Antisocial Personality & $0.30^{*}$ & $0.26^{*}$ \\
\hline
\end{tabular}

Note. IA = Impulsive Aggression; PA = Premeditated Aggression; BPAQ = Buss-Perry Aggression Questionnaire; BIS-11 = Barratt Impulsiveness Scale; Psychopathic Personality Inventory = PPI; Psychopathy Checklist—revised = PCL-R; Eysenck Personality Inventory = EPQ; Lifetime History of Aggression = LHA; State-Trait Anger $\quad$ Expression Inventory = STAXI; NEO-Personality Inventory-Revised = NEO-PI-R; Youth Self-Report Syndrome = YSR; Reactive Proactive Questionnaire = RPQ; Personality Assessment Inventory = PAI; Barratt Impulsiveness Scale- Brief Version = BIS-Brief; Difficulties with Emotion Regulation Scale = DERS; Self-Report of Aggression and Social Behavior Measure = SRASBM; Personality Diagnostic Questionnaire-4 Cluster B = PDQ-4.

${ }^{*} p \leq .05$.

** $p<.01$.

et al., 2013; Cruz \& Barbosa, 2010) and investigating the association between IPAS and other aggression related constructs (e.g., Haden et al., 2008; Steinberg et al., 2013; Teten Tharp et al., 2011), as well as IPAS and childhood maltreatment and negative life experiences (Chen et al., 2013; Haden et al., 2008). Among community samples the scale demonstrated strong internal consistency, a factorial structure comparable to previous studies, constant across men and women, and evidence of convergent validity with other aggression scales. It was also found that IA is the predominant pattern among community samples (Cruz et al., 2019; Cruz \& Barbosa, 2010; Haden et al., 2008; Rodríguez, 2015). Research on community samples have also demonstrated the predictive value of the association between impulsive and premeditated aggression and personality disorders in non-clinical samples (Chen et al., 2013; Ostrov \& Houston, 2008). The previous results reinforce the importance of addressing aggressive functions to inform risk assessment and violence prevention programs (e.g., Haden et al., 2008).

\subsubsection{Veterans suffering from PTSD}

Impulsivity is a characteristic of individuals suffering from trauma (Teten et al., 2010). Therefore, IPAS was used to examine the two features of aggression in veterans in association with aggression related constructs and emotional process deficits (Teten et al., 2008; Teten Tharp et al., 2011). Similar to community samples, IA was the predominant form of aggressive behavior, and significantly predicted a diagnosis of PTSD among veterans (Teten et al., 2010). Increasing the knowledge about the value of IA and PA aggression in PTSD will improve treatment strategies for this group of individuals.

\subsubsection{Substance use disorders}

Impulsivity is reported in substance users (Stanford et al., 2009). The relation between patterns of aggression and substance abuse was addressed in community samples with psychopathic traits and antisocial personality disorders (Alcorn et al., 2013; Long et al., 2014) and suicidal behavior (Conner et al., 2009). Few studies have reported the relation between aggression, suicidal ideation and attempt in substance users' samples. In a sample of drug users PA demonstrated particular relevance in the explanation of suicidal behaviors in male and female individuals (Conner et al., 2009). It is suggested that emotion regulation acts as a mediator between substance abuse and psychopathy (Long et al., 2014). In addition, the comorbidity between antisocial personality disorder and substance use disorder increases the probability of higher aggression and particularly the risk for impulsive dysregulation (Alcorn et al., 2013).

Similar to community samples without substance use, the scale appears to be stable across gender and reliable across time (Conner et al., 2007) with reports of high internal consistency (e.g., Conner et al., 2007; Long et al., 2014). Accordingly, the assessment of IA and PA features would greatly inform treatment strategies delivered to drug addicts.

\subsubsection{Samples with psychopathology or personality disorders}

The IPAS has been administered in a notable range of clinical populations (e.g. schizophrenic patients, individuals with affective, anxiety and psychotic disorders, adolescents with conduct disorder), differentiating impulsive from premeditated individuals (Mathias et al., 2007; Romans et al., 2015). These studies focused on the importance of assessing aggressive features to better inform risk, management and treatment services.

The IPAS has been employed in physical aggressive men (Stanford et al., 2003) to characterize the participants' primarily aggressive behavior as either impulsive or premeditated. Strong reliability and validity were reported for the IPAS, and IA was the predominant pattern of aggression in $90 \%$ of individuals.

Schizophrenic patients have also been assessed with the IPAS. The results demonstrated a positive association between PA and psychopathic features, and antisocial personality disorder, in patients with schizophrenia (Bo et al., 2013a, 2013b). Increased psychopathic features and PA are also associated with diminished mentalizing abilities (cognitive and affective processes important for interpersonal regulation) (Bo, Abu-Akel, Kongerslev, Haahr, \& Bateman, 2014).

Likewise, subtypes of aggressive behavior were evaluated in adolescents with conduct disorder from the community. Results showed 
good psychometric properties of the IPAS scales (Mathias et al., 2007) and individual differences according to their predominant aggressive pattern in conduct disorder adolescents (Gauthier et al., 2009). Impulsive adolescents revealed impaired emotional, thought, social and personality functions (Mathias et al., 2007) and were described as more socially-detached and expressing negative emotions (Gauthier et al., 2009), while high PA adolescents were described as manipulative and egocentric (Gauthier et al., 2009). Time perception (ability to estimate a 1-min interval) of impulsive adolescents with conduct disorder was significantly shorter when compared to premeditated adolescents with conduct disorder or healthy controls (Dougherty et al., 2007). Research in time estimation processes suggest that accuracy in these tasks is more dependent on the subtype of aggression than on the history of previous or current violent or non-violent incidents or antisocial acts (Dougherty et al., 2007). This is important because it reveals information about an individual ability for self-regulation, that when impaired compromises its actions and can be a risk factor for aggressive and violent behaviors.

All the above reinforce the need of addressing subtypes of aggression in samples with psychopathology or personality disorders to improve clinical interventions and treatment compliance.

The IPAS scale was also employed to categorize between impulsive and premeditated aggression in participants undergoing pharmacological treatment for chronic aggressive behavior (Houston \& Stanford, 2006). The study concluded that IA and PA features are important in treatment adherence revealing that those who did not respond successfully to the pharmacological treatment endorsed more premeditated as opposed to impulsive aggressive behavior characteristics (Houston \& Stanford, 2006).

\subsubsection{Forensic populations}

Aggression, violence and crime are intertwined constructs. Nevertheless, literature has been pointing out the difficulty to disentangling offenders' characteristics to address intervention needs. Accordingly, the IPAS has been shown to be a valuable tool in forensic settings to assess premeditation and impulsivity.

Steadham and Rogers (2013) used the IPAS to classify detainees on a minimum-security prison, demonstrating that the scale is also useful in less severe contexts and that risk assessment benefits from information not only about the type of aggression but also its link with personality traits (e.g., antisocial personality disorder, conduct disorder and psychopathy). It was also found that premeditation was a predictor of violent recidivism (Swogger, Walsh, Christie, et al., 2014). This longitudinal study has demonstrated the importance of assessing aggressive tendencies to increase the sensitivity of re-offending treatment.

Swogger et al. (2010) have employed the IA/PA classification to help determining the relation between aggression, psychopathy and Axis I disorders, reporting an association between premeditation and psychopathy and impulsivity and psychopathology. The results have implications on risk assessment, management and treatment within criminal samples and replicate previous findings on community samples (e.g., Bo et al., 2013a, 2013b; Gauthier et al., 2009; Mathias et al., 2007).

The IPAS was also used to compare aggressive tendencies in domestic violence offenders and successfully classified male offenders as premeditated or impulsive (Stanford et al., 2008). Similar to previous results on aggression and personality related features, perpetrators of intimate partner violence with higher premeditated scores revealed higher results on psychopathic traits, as well as in treatment rejection and compliance. Impulsive batterers reported a wider range of psychopathology. These findings highlight the importance of adapting intervention to an individual aggressive pattern to increase programs efficacy (Stanford et al., 2008). Lake and Stanford (2011) replicated the previous study in a sample of female perpetrators of intimate partner violence and have found that IA was the predominant pattern of aggression on this sample. They have also demonstrated differences for the male batterers results, since women have the same odds to comply with treatment more successfully, regardless the aggressive pattern.

In forensic settings the discrimination between IA and PA has also proved to be useful to tackle suicide risk. Comparable to data reported in community samples, IA offenders are at higher risk of attempting suicide, therefore preventive intervention would benefit from assessment tools to characterize offenders as either premeditated or impulsive (Swogger, Walsh, Maisto, \& Conner, 2014).

\section{Discussion and conclusions}

The key findings from this review are in support of the IPAS being a useful tool to discriminate between impulsive and premeditated aggression both in clinical, forensic and non-clinical settings (such as educational context). This study aimed to present evidences of IPAS psychometric properties within different samples and the scale versatility among samples and different cultural settings. It is noteworthy from this review that the internal consistency of the IPAS appears to be good, regarding the reported sample scores. Internal consistency (Cronbach's alpha) of both the IA and PA scales falls within an acceptable to excellent range (IA: 0.68-0.93; PA: 0.70-0.90). In addition, reliability generalization analysis demonstrated no significant differences between sample characteristics nor language, suggesting that the scale is reliable across different cultures and with clinical or non-clinical samples. This result supports its use whether for clinical or forensic populations referred for aggression-related behaviors, or communitydwelling individuals. We should also consider that the effect sizes, namely the ones for IA scales, would benefit from an increase in the number of studies. That would help to clarify the reliability generalization of the scores. Even with limited data, it is suggested that the dimensional scoring might be more accurate than the categorical procedure to identify impulsive features, which is consistent with this scoring technique that accounts for the predominant form of aggressiveness rather than a category of aggressive behaviors.

Construct and concurrent validity for the IPAS scales has been supported with small to moderate significant relationships between different personality and aggression measures, corroborating the relation between impulsive and premeditated aggression with similar selfreport measures of aggression, aggressive related constructs and personality characteristics.

Moreover, regarding the different samples assessed, the IPAS demonstrated the presence of two types of aggressive behavior - impulsive and premeditated, accurately characterizing individuals as either impulsive or premeditated. Although the two-dimensional classification of aggression accounts for both IA and PA forms, across samples it was demonstrated that IA is the most predominant pattern of aggression.

Assessing aggression either as predominantly impulsive or premeditated explains the tendency in forensic samples for these two scales to be significantly correlated, suggesting that offender groups present both subtypes. This may be due to criminal versatility (Kockler et al., 2006), as these "extremes are 'ideal types' that seldom occur in pure form" (Block \& Block, 1992, cit in. Kockler et al., 2006). This also could be because although one of the subtypes of aggression is predominant, the other can also be present, but to a less extent. Both dimensional and categorical approaches provide useful information, and account for the natural heterogeneity of aggressive behavior, recognizing that individuals may vary in their level of behavioral control, having, however, a predominance for one type of aggression (Houston \& Stanford, 2006). Other possible explanation concerns a response bias commonly affecting both scales, as antisocial participants tend to project more social desirability when answering self-report questionnaires (Barbosa, Almeida, Ferreira-Santos, \& Marques-Teixeira, 2015).

Aggressive behaviors are disruptive and may constitute a risk for self and others. They are often present in samples that have suffered from post-traumatic stress disorder such as veterans, individuals with a history of substance abuse or personality disorders. From a clinical perspective we should consider the importance of knowing and 
predicting the individual predominant form of aggression to increase individual responsivity and treatment efficacy. By assessing the individual predominant form of aggression, IPAS can also inform on risk and management strategies and help to prevent misconduct for self and others.

IPAS requires minimal training for assessment and can be utilized on both forensic and community samples, for clinical and research purposes. Both research and practice benefit from a measure that reveals good psychometric properties, is translated and validated in several countries and different settings, easy to apply and with demonstrated correlation with other aggression-related and personality measures.

Using IPAS to characterize aggressive behavior has implications on the definition and implementation of violence and re-offending prevention, treatment and intervention programs (Haden et al., 2008; Meijers, Harte, Jonker, \& Meynen, 2015). For example, in a research conducted with mainly male individuals with high PA scores it was reported that they did not engage or successfully respond to treatment in comparison with impulsive individuals (Houston \& Stanford, 2006). Risk assessment with potentially aggressive individuals benefits from this more nuanced view of aggression provided by IPAS, regardless of being a forensic or a community sample. Better assessment promotes more effective and adequate intervention and treatment which in turn increases levels of adherence and compliance of the participants.

There are also limitations concerning the scale, namely the already mentioned debate on which the best approach to scoring IPAS is. It is noteworthy that most studies adopted the categorical scoring technique, but there is also evidence supporting the dimensional scoring technique, since it could offer more accurate information on the magnitude of aggressiveness and differences between IA and PA offenders (Steadham \& Rogers, 2013).

In conclusion, IPAS shows good psychometric properties in terms of item characteristics and acceptable to good reliability across samples with results suggesting that the scale reliability could be generalized regarding sampling scoring, version (either English or translated) and sample settings. Future research should replicate present findings to establish more robust comparisons and associations between impulsivity and premeditation and other related constructs accounting for both aggressive and non-aggressive samples.

Researchers should be aware that since impulsivity and premeditation are not mutually exclusive dimensions of aggression. Future studies should combine, in the characterization of the respondent as predominantly impulsive or predominantly premeditated, additional measures such as observational and behavioral data, informant and police reports, and clinical files, when available.

\section{Funding}

This work was supported by The Foundation for Science and Technology (Grant number SFRH/BD/76062/2011), and the Portuguese Ministry of Science, Technology and Higher Education through national funds and co-financed by FEDER and COMPETE2020 under the PT2020 Partnership Agreement (POCI-01-0145-FEDER007653) - Psychology Research Centre (UID/PSI/01662), awarded to the first author. The study was also supported by Grant SFRH/BPD/ 108602/2015 from the Portuguese Foundation for Science and Technology awarded to Andreia de Castro-Rodrigues.

\section{Declarations of interest}

None.

\section{References}

Alcorn, J. L., Gowin, J. L., Green, C. E., Swann, A. C., Moeller, F. G., \& Lane, S. D. (2013). Aggression, impulsivity, and psychopathic traits in combined antisocial personality disorder and substance use disorder. Journal of Neuropsychiatry and Clinical Neurosciences, 25(3), 229-232. https://doi.org/10.1176/appi.neuropsych.12030060.

Azevedo, J. C., Pais-Ribeiro, J. L., Coelho, R., \& Figueiredo-Braga, M. (2018). Validation of the Portuguese version of impulsive-premeditated aggression scale in an inmate population. Frontiers in Psychiatry, 9(FEB), 1-8. https://doi.org/10.3389/fpsyt.2018. 00010.

Barbosa, F., Almeida, P. R., Ferreira-Santos, F., \& Marques-Teixeira, J. (2015). Using signal detection theory in the analysis of emotional sensitivity of male recidivist offenders. Criminal Behaviour and Mental Health. https://doi.org/10.1002/cbm.1950 n/ a-n/a.

Barratt, E., Stanford, M., Felthous, A., \& Kent, T. (1997). The effects of phenytoin on impulsive and premeditated aggression: A controlled study. Journal of Clinical Psychopharmacology, 17(5), 341-349.

Block, R., \& Block, C. R. (1992). Homicide syndromes and vulnerability: Violence in Chicago community areas over 25 years. Studies on Crime \& Crime Prevention, 1, 61-87.

Bo, S., Abu-Akel, A., Kongerslev, M., Haahr, U. H., \& Bateman, A. (2014). Mentalizing mediates the relationship between psychopathy and type of aggression in schizophrenia. The Journal of Nervous and Mental Disease, 202(1), 55-63. https://doi.org/ 10.1097/NMD.0000000000000067.

Bo, S., Forth, A., Kongerslev, M., Haahr, U. H., Pedersen, L., \& Simonsen, E. (2013a). Subtypes of aggression in patients with schizophrenia: The role of personality disorders. Criminal Behaviour and Mental Health, 23(2), 124-137. https://doi.org/10. 1002/cbm.1858.

Bo, S., Forth, A., Kongerslev, M., Haahr, U. H., Pedersen, L., \& Simonsen, E. (2013b). Subtypes of aggression in patients with schizophrenia: The role of psychopathy. The Journal of Forensic Psychiatry and Psychology, 24(4), 496-513. https://doi.org/10. 1002/cbm.1858.

Bushman, B. J., \& Anderson, C. A. (2001). Is it time to pull the plug on the hostile versus instrumental aggression dichotomy? Psychological Review, 108(1), 273-279. https:// doi.org/10.1037/0033-295X.108.1.273.

Chen, F. R., Yang, Y., \& Qian, M. (2013). Chinese version of impulsive/premeditated aggression scale: Validation and its psychometric properties. Journal of Aggression, Maltreatment \& Trauma, 22(2), 175-191. https://doi.org/10.1080/10926771.2013. 741664.

Conner, K. R., Houston, R. J., Sworts, L. M., \& Meldrum, S. (2007). Reliability of the impulsive-premeditated aggression scale (IPAS) in treated opiate-dependent individuals. Addictive Behaviors, 32(3), 655-659. https://doi.org/10.1016/j.addbeh. 2006.06.026.

Conner, K. R., Swogger, M. T., \& Houston, R. J. (2009). A test of the reactive aggressionsuicidal behavior hypothesis: Is there a case for proactive aggression? Journal of Abnormal Psychology, 118(1), 235-240. https://doi.org/10.1037/a0014659.

Cruz, A. R., \& Barbosa, F. (2010). Contribution to the characterization of aggressive behvior - an exploratory study based on the impulsive-premeditated aggression scale.pdf. Psiquiatria, Psicologia e Justiça, 3.

Cruz, A. R., Pasion, R., Rodrigues, A., Zabala, C., Ricarte, J., \& Barbosa, F. (2019). Psychometric properties of the impulsive/premeditated aggression scale in Portuguese community and forensic samples. Trends in Psychiatry and Psychotherapy. https://doi.org/10.1590/2237-6089-2018-0055.

Derkzen, D. M. (2007). Impulsivity, social problem solving and alcohol dependency as contributors to aggression in a sample of provincially incarcerated offenders. (Saskatchewan, Saskatoon).

Dougherty, D. M., Dew, R. E., Mathias, C. W., Marsh, D. M., Addicott, M. A., \& Barratt, E. S. (2007). Impulsive and premeditated subtypes of aggression in conduct disorder: Differences in time estimation. Aggressive Behavior, 33(6), 574-582. https://doi.org/ 10.1002/ab.20219.

Gauthier, K. J., Furr, R. M., Mathias, C. W., Marsh-Richard, D. M., \& Dougherty, D. M. (2009). Differentiating impulsive and premeditated aggression: Self and informant perspectives among adolescents with personality pathology. Journal of Personality Disorders, 23(1), 76-84. https://doi.org/10.1521/pedi.2009.23.1.76.

Haden, S. C., Scarpa, A., \& Stanford, M. S. (2008). Validation of the impulsive/premeditated aggression scale in college students. Journal of Agression, Maltreatment \& Trauma, 17(3), 352-373. https://doi.org/10.1080/10926770802406783.

Houston, R. J., \& Stanford, M. S. (2006). Characterization of aggressive behavior and phenytoin response. Aggressive Behavior, 32, 38-43. https://doi.org/10.1002/ab. 20104.

Kockler, T. R., Stanford, M. S., Nelson, C. E., Meloy, J. R., \& Sanford, K. (2006) Characterizing aggressive behavior in a forensic population. The American Journal of Orthopsychiatry, 76(1), 80-85. https://doi.org/10.1037/0002-9432.76.1.80.

Kuyck, W. G. E., de Beurs, E., Barendregt, M., \& van den Brink, W. (2013). Psychometric evaluation of the Dutch version of the Impulsive/Premeditated Aggression Scale (IPAS) in male and female prisoners. International Journal of Forensic Mental Health, 12(3), 172-179. https://doi.org/10.1080/14999013.2013.819396.

Lake, S. L., \& Stanford, M. S. (2011). Comparison of impulsive and premeditated female perpetrators of intimate partner violence. Partner Abuse, 2(3), 284-299. https://doi. org/10.1891/1946-6560.2.3.284.

Long, K., Felton, J. W., Lilienfeld, S. O., \& Lejuez, C. W. (2014). The role of emotion regulation in the relations between psychopathy factors and impulsive and premeditated aggression. Personality Disorders, Theory, Research, and Treatment, 5(4), 390-396. https://doi.org/10.1037/per0000085.

Mathias, C. W., Stanford, M. S., Marsh, D. M., Frick, P. J., Moeller, F. G., Swann, A. C., \& Dougherty, D. M. (2007). Characterizing aggressive behavior with the Impulsive/ Premeditated Aggression Scale among adolescents with conduct disorder. Psychiatry Research, 151(3), 231-242. https://doi.org/10.1016/j.psychres.2006.11.001.

Meijers, J., Harte, J. M., Jonker, F. A., \& Meynen, G. (2015). Prison brain? Executive dysfunction in prisoners. Frontiers in Psychology, 6. https://doi.org/10.3389/fpsyg 
2015.00043.

Meloy, J. R. (2006). Empirical basis and forensic application of affective and predatory violence. The Australian and New Zealand Journal of Psychiatry, 40(6-7), 539-547. https://doi.org/10.1111/j.1440-1614.2006.01837.x.

Moeller, F. G., Barratt, E. S., Dougherty, D. M., Schmitz, J. M., \& Swann, A. C. (2001). Psychiatric aspects of impulsivity. American Journal of Psychiatry, 158(11), 1783-1793. https://doi.org/10.1176/appi.ajp.158.11.1783.

Ostrov, J. M., \& Houston, R. J. (2008). The utility of forms and functions of aggression in emerging adulthood: Association with personality disorder symptomatology. Journal of Youth and Adolescence, 37(9), 1147-1158. https://doi.org/10.1007/s10964-0089289-4.

Parrott, D. J., \& Giancola, P. R. (2007). Addressing "The criterion problem" in the assessment of aggressive behavior: Development of a new taxonomic system. Aggression and Violent Behavior, 12(3), 280-299. https://doi.org/10.1016/j.avb.2006.08.002.

Raine, A., Meloy, J. R., Bihrle, S., Stoddard, J., LaCasse, L., \& Buchsbaum, M. S. (1998). Reduced prefrontal and increased subcortical brain functioning assessed using positron emission tomography in predatory and affective murderers. Behavioral Sciences \& the Law, 16(3), 319-332. Retrieved from http://www.ncbi.nlm.nih.gov/pubmed/ 9768464.

Rodríguez, L. (2015). Validación psicométrica de la versión española de la Escala de Agresión Impulsiva y Premeditada (IPAS). Universidad Complutense de Madrid - Faculdad de Psicologiía.

Rodriguez, M. C., \& Maeda, Y. (2006). Meta-analysis of coefficient alpha. Psychological Methods, 11(3), 306-322. https://doi.org/10.1037/1082-989X.11.3.306.

Romans, L., Fresán, A., Sentíes, H., Sarmiento, E., Berlanga, C., Robles-García, R., \& Tovilla-Zarate, C.-A. (2015). Validation of the Impulsive/Premeditated Aggression Scale in Mexican psychiatric patients. Nordic Journal of Psychiatry, 69(5), 397-402. https://doi.org/10.3109/08039488.2014.994033.

Rouse, S. V. (2007). Using reliability generalization methods to explore measurement error: An illustration using the MMPI-2 PSY-5 scales. Journal of Personality Assessment, 88(3), 264-275. https://doi.org/10.1080/00223890701293908.

Stanford, M. S. (n.d.). Classification procedures. Unpublished manual.

Stanford, M. S., Houston, R. J., \& Baldridge, R. M. (2008). Comparison of impulsive and premeditated perpetrators of intimate partner violence. Behavioral Sciences \& the Law, 26(6), 709-722. https://doi.org/10.1002/bsl.808.

Stanford, M. S., Houston, R. J., Mathias, C. W., Villemarette-Pittman, N. R., Helfritz, L. E., \& Conklin, S. M. (2003). Characterizing aggressive behavior. Assessment, 10(2), 183-190. Retrieved from http://www.ncbi.nlm.nih.gov/pubmed/12801190.

Stanford, M. S., Mathias, C. W., Dougherty, D. M., Lake, S. L., Anderson, N. E., \& Patton, J. H. (2009). Fifty years of the Barratt impulsiveness scale: An update and review. Personality and Individual Differences, 47(5), 385-395. https://doi.org/10.1016/j. paid.2009.04.008.

Steadham, J. A., \& Rogers, R. (2013). Predictors of reactive and instrumental aggression in jail detainees: An initial examination. Journal of Forensic Psychology Practice, 13(5), 411-428. https://doi.org/10.1080/15228932.2013.847350.
Steinberg, L., Sharp, C., Stanford, M. S., \& Tharp, A. T. (2013). New tricks for an old measure: The development of the Barratt impulsiveness scale-brief (BIS-brief). Psychological Assessment, 25(1), 216-226. https://doi.org/10.1037/a0030550.

Suris, A., Lind, L., Emmett, G., Borman, P. D., Kashner, M., \& Barratt, E. S. (2004). Measures of aggressive behavior: Overview of clinical and research instruments. Aggression and Violent Behavior, 9(2), 165-227. https://doi.org/10.1016/S1359. 1789(03)00012-0.

Swing, E. L., \& Anderson, C. A. (2014). The role of attention problems and impulsiveness in media violence effects on aggression. Aggressive Behavior, 40(3), 197-203. https:// doi.org/10.1002/ab.21519.

Swogger, M. T., Walsh, Z., Christie, M., Priddy, B. M., \& Conner, K. R. (2014). Impulsive versus premeditated aggression in the prediction of violent criminal recidivism. Aggressive Behavior, 41(4), 346-352. https://doi.org/10.1002/ab.21549.

Swogger, M. T., Walsh, Z., Houston, R. J., Cashman-Brown, S., \& Conner, K. R. (2010). Psychopathy and Axis I psychiatric disorders among criminal offenders: Relationships to impulsive and proactive aggression. Aggressive Behavior, 36(1), 45-53. https://doi. org/10.1002/ab.20330.

Swogger, M. T., Walsh, Z., Maisto, S. A., \& Conner, K. R. (2014). Reactive and proactive aggression and suicide attempts among criminal offenders. Criminal Justice and Behavior, 41(3), 337-344. https://doi.org/10.1177/0093854813508764.

Teten, A. L., Miller, L. A., Bailey, S. D., Dunn, N. J., \& Kent, T. A. (2008). Empathic deficits and alexithymia in trauma-related impulsive aggression. Behavioral Sciences \& the Law, 26(6), 823-832. https://doi.org/10.1002/bsl.843.

Teten, A. L., Miller, L. A., Stanford, M. S., Petersen, N. J., Bailey, S. D., Collins, R. L., ... Kent, T.a. (2010). Characterizing aggression and its association to anger and hostility among male veterans with post-traumatic stress disorder. Military Medicine, 175(6), 405-410.

Teten Tharp, A. L., Sharp, C., Stanford, M. S., Lake, S. L., Raine, A., \& Kent, T.a. (2011) Correspondence of aggressive behavior classifications among young adults using the impulsive premeditated aggression scale and the reactive proactive questionnaire. Personality and Individual Differences, 50(2), 279-285. https://doi.org/10.1016/j paid.2010.10.003.

Vassar, M., \& Bradley, G. (2010). A reliability generalization study of coefficient alpha for the life orientation test. Journal of Personality Assessment, 92(4), 362-370. https://doi. org/10.1080/00223891.2010.482016.

Vitaro, F., Brendgen, M., \& Tremblay, R. E. (2002). Reactively and proactively aggressive children: Antecedent and subsequent characteristics. Journal of Child Psychology and Psychiatry, and Allied Disciplines, 43(4), 495-505. https://doi.org/10.1111/1469. 7610.00040

Volavka, J. (1999). The neurobiology of violence: An update. The Journal of Neuropsychiatry and Clinical Neurosciences, 11(3), 307-314.

Wilson, L. C., \& Scarpa, A. (2014). Aggressive behavior: An alternative model of resting heart rate and sensation seeking. Aggressive Behavior, 40(1), 91-98. https://doi.org/ 10.1002/ab.21504. 